\title{
Histoire d'une institution au cœur de la Francophonie
}

Michèle Gendreau-Massaloux

\section{(2) OpenEdition \\ 12 Journals}

\section{Édition électronique}

URL : https://journals.openedition.org/dhfles/119

DOI : $10.4000 /$ dhfles. 119

ISSN : 2221-4038

Éditeur

Société Internationale pour l'Histoire du Français Langue Étrangère ou Seconde

Édition imprimée

Date de publication : 1 janvier 2008

Pagination : 121-130

ISSN : 0992-7654

\section{Référence électronique}

Michèle Gendreau-Massaloux, "Histoire d'une institution au cœur de la Francophonie », Documents

pour l'histoire du français langue étrangère ou seconde [En ligne], 40/41 | 2008, mis en ligne le 17

décembre 2010, consulté le 27 mai 2021. URL : http://journals.openedition.org/dhfles/119; DOI :

https://doi.org/10.4000/dhfles.119

Ce document a été généré automatiquement le 27 mai 2021.

(C) SIHFLES 


\title{
Histoire d'une institution au cœur de la Francophonie
}

\author{
Michèle Gendreau-Massaloux
}

1 Je vous remercie de faire appel à une universitaire devenue, par élection, gestionnaire d'une institution de la Francophonie, l'Agence universitaire de la Francophonie (AUF). Comme vous le savez, l'AUF tente de maintenir avec votre association des relations permanentes, grâce au suivi actif de Monsieur Patrick Chardenet, en poste à Montréal, qui a pris l'habitude de se tenir informé de vos activités, et notamment des manifestations que vous préparez. Celle-ci nous est apparue, comme les autres, riche de communications pertinentes, parce que portant sur des thèmes rarement abordés, et je suis ravie d'y participer.

2 Je reviendrai sur l'histoire de l'AUF, institution universitaire et francophone. Ces deux dimensions sont indissociables: la Francophonie universitaire doit beaucoup au numéro de novembre 1962 de la revue Esprit, sous-titrée «Le français, langue vivante ", et s'avère, de la sorte, fortement tributaire des mouvements idéologiques et politiques qui ont conduit à l'émergence d'une Francophonie institutionnelle, aujourd'hui incarnée par son secrétaire général Abdou Diouf. Quant à la dimension universitaire, elle a permis à l'AUF d'affirmer, depuis ses débuts, son originalité à l'égard des autres composantes de la Francophonie institutionnelle.

3 L'histoire de l'AUF, et notamment les dates symboliques qui ont marqué les étapes successives de sa création, reste, en premier lieu, indiscutablement liée à celle de la Francophonie institutionnelle. Ainsi, l'année 1962 marque un tournant. Trois événements se produisent : tout d'abord l'apparition d'une volonté de coopération, qui se concrétisera un an plus tard par la création de l'ACCT (Agence de coopération culturelle et technique), dont Niamey sera la souche; ensuite, l'affirmation par le Québec d'un choix culturel, son caractère «francophone", et sa revendication d'entité politique autonome au sein de l'ensemble canadien; enfin, la prise de position de Léopold Sédar Senghor, Habib Bourguiba, Amani Diori et Norodom Sihanouk en faveur du français, prise de position relayée notamment par la presse québécoise et, au niveau universitaire, par deux personnalités, André Bachand, alors directeur des relations 
extérieures de l'université de Montréal, et Jean-Marc Léger, journaliste au quotidien montréalais Le Devoir. La conjonction de ces trois réalités est à l'origine d'un mouvement de solidarité international entre les pays considérant le français comme l'une de leurs langues nationales. Si vous souhaitez en savoir davantage sur les conditions de l'émergence de la Francophonie politique, qui ont évidemment eu des incidences sur le mouvement universitaire francophone, je me permets de vous renvoyer à la lettre numéro 9 de l'Institut François Mitterrand, publiée en septembre 2004, dans laquelle je reviens sur ces questions. Je rappellerai seulement ici que, si la Francophonie politique a tardé à se concrétiser, elle doit en partie ce retard au refus systématique de Pierre Elliot Trudeau, alors Premier ministre du gouvernement fédéral d'Ottawa, de siéger, lors des conférences internationales, à la même table que René Lévesque, Premier ministre souverainiste du Québec, qu'il refusait de considérer comme son « homologue ». L'élection de Martin Brian Mulroney, en 1984, à la tête du gouvernement canadien a, heureusement, changé cette donne et permis de réunir, pour la première fois, en mars 1986, un Sommet politique de la Francophonie.

4 Mais revenons à l'histoire proprement universitaire du mouvement francophone. Dans les années 60, un certain nombre d'universitaires, parmi lesquels des membres de l'Église catholique - dont Monseigneur Irénée Lussier, recteur de l'université de Montréal -, se fondant sur leur désir commun de voir le français reconnu comme une langue d'enseignement supérieur et de recherche, ont dessiné les contours d'un réseau universitaire associant des pays emblématiques, comme le Sénégal, le Niger ou la Tunisie, qui se constituera rapidement, avec le soutien engagé du Québec et du Canada, en association. En 1961, Monseigneur Irénée Lussier, Lucien Paye, recteur de la toute jeune université de Dakar, et Robert Mallet, mon prédécesseur au poste de chancelier des universités de Paris, ont ainsi, avec quelques autres, fondé l'association des universités partiellement ou entièrement de langue française (AUPELF). Cette dimension associative est fondamentale, car elle apporte à la fois la garantie d'une liberté et le risque d'une fragilité. Nous savons tous en effet combien il est difficile d'assurer la pérennité des ressources budgétaires d'une association. L'AUPELF ne vivait que des cotisations des établissements membres. Cependant, une partie de ces membres, notamment en Afrique, entretenaient avec les États des relations de forte proximité, et portaient une légitimité politique en même temps qu'une réflexion sur les composantes du monde universitaire. Il faut souligner, à cet égard, que les recteurs des universités de nombre de pays d'Afrique étaient nommés par leur gouvernement, à la différence, par exemple, des présidents d'université français, élus par leurs pairs. Cette proximité avec le politique constituait sans doute une chance, mais elle comportait également un risque en termes d'autonomie de décision.

5 Lors de l'Assemblée générale de 1972, à l'initiative du recteur Mallet, la France, jusquelà en retrait par rapport à l'engagement initial des Africains et des Québécois, s'investit à son tour dans ce mouvement associatif, dont le recteur Mallet est alors nommé président. Il s'en fera le porte-parole enflammé et restera très fier de cette nomination. Le réseau compte, à cette époque, quarante universités membres, issues d'une dizaine d'États qui commencent à s'interroger sur la possibilité de trouver des financements complémentaires pour cette jeune institution qu'est l'AUPELF. Un programme commun est défini, déjà fondé sur le souci de garantir aux universités du Sud des systèmes d'équivalence avec les diplômes délivrés par celles du Nord : dans les années 70 , rares sont en effet les Africains qui peuvent concourir depuis leur pays à l'agrégation ou soutenir une thèse d'État; ils sont donc contraints à de longs séjours à l'étranger, au 
Canada, en Belgique, en France ou en Suisse. On mesurera mieux le chemin parcouru en remarquant qu'aujourd'hui les pôles d'excellence situés au Sud, non seulement permettent aux étudiants de passer ces diplômes en recevant un titre équivalent à celui d'un pays du Nord, mais l'AUF commence, en outre, à dispenser des enseignements à distance entièrement conçus au Sud.

6 Au sein de l'AUPELF, la coopération universitaire est donc première, et non la langue, ce qui apparaît clairement dans les statuts de l'association. Une instance suprême se réunit tous les trois ans. Cette Assemblée générale, qui regroupe les représentants des établissements membres, élit un Conseil d'administration constitué d'un président, de deux vice-présidents et de six membres, Conseil chargé de mettre en œuvre une politique définie collégialement. Dans cette assemblée d'universités francophones, les universitaires sont donc les décideurs. Ce signe fort, constitutif, perdure aujourd'hui, au prix d'un combat que j'ai eu moi-même à mener.

7 A la suite de Monseigneur Lussier, un président issu du Maghreb est élu recteur de l'AUPELF, Mohamed El Fasi, figure emblématique, grand érudit marocain qui a donné son nom à l'un des premiers prix scientifiques décerné par l'AUPELF pour récompenser l'œuvre scientifique d'un chercheur qui publie en français.

8 En même temps que l'association vit, ou plutôt vivote, le mouvement politique se développe. L'ACCT, dont les activités concernent principalement la culture et l'enseignement primaire et secondaire, porte un intérêt réel au développement de l'AUPELF. Lors du Sommet des chefs d'État et de gouvernement ayant en commun l'usage du français qui se réunit à Québec, en 1987, un projet de transformation de l'AUPELF est présenté : il s'agit d'adjoindre à l'association une « université des réseaux d'expression française» (UREF). Si cette dénomination, ambiguë, pose des questions aux responsables d'universités «réelles", qui ne voient pas bien la fonction de cette " université sans mur ", elle est néanmoins adoptée. On parlera désormais de l'AUPELFUREF. La dualité inscrite dans ce nouveau sigle entretient rapidement une certaine confusion; les programmes, en particulier, sont trop nombreux. Progressivement, l'intérêt des États faiblit face au manque d'indicateurs de performance et d'efficacité. Les dépenses de personnels s'avèrent en outre excessives; le poids des frais généraux par rapport au budget alloué aux programmes peu soutenable. Une crise financière s'installe, qui devient une crise de confiance. Les chefs d'État et de gouvernement réunis au Sommet de Moncton, en 1999, décident de remplacer le recteur en poste et réclament la rédaction de nouveaux statuts.

9 Se pose alors un choix crucial quant à la position que doit adopter l'association vis-à-vis des États, choix rendu plus difficile par l'évolution récente de l'AUPELF. Dès le Sommet de Dakar en 1989, l'institution universitaire, consciente des limites de ses moyens financiers, s'est en effet rapprochée de la Francophonie politique. En accord avec les chefs d'État et de gouvernement, l'AUPELF a adopté le statut d'opérateur de la Francophonie. Lors du Sommet de Hanoi, en 1997, le schéma de la Francophonie institutionnelle s'est encore compliqué : Boutros Boutros-Ghali a été nommé au poste de Secrétaire général d'un Conseil permanent qui adoptera, en 1998, l'appellation d'Organisation internationale de la Francophonie, et Abdou Diouf, ancien Président du Sénégal, lui succède en 2002. Autour du Secrétaire général agissent divers opérateurs : l'ACCT, qui deviendra l'Agence intergouvernementale de la Francophonie (AIF) en 1999 ; l'AUPELF-UREF, qui deviendra l'Agence universitaire de la Francophonie en 2001 ; TV5 ; l'Association internationale des maires francophones (AIMF) ; l'Université 
Senghor d'Alexandrie... Ce montage complexe permet à l'AUPELF-UREF de bénéficier non seulement des cotisations de ses universités membres, mais également des financements des États. En contrepartie, l'association devient tributaire des décisions politiques de la Francophonie institutionnelle qui peut, le cas échéant, lui confier des missions gouvernementales. Lors de la crise financière de 1998, avant la rédaction des nouveaux statuts de l'association, une question fondamentale se pose donc, qui implique l'ensemble des partenaires, c'est-à-dire les Français, les Canadiens, les Québécois, les Belges, mais aussi les Africains et les représentants d'Asie et d'Europe centrale et orientale : l'AUPELF-UREF doit-elle devenir un «double » universitaire de l'AIF piloté par les États - position du gouvernement français - ou bien doit-elle conserver son caractère universitaire et son autonomie de décision? Dans le débat qui s'instaure entre les diverses parties prenantes, je plaide pour que l'institution conserve une identité universitaire et que soit confirmée la prévalence des universités au sein du Conseil d'administration.

10 Je passe sous silence les épisodes, nombreux, qui conduisent à la rédaction définitive des nouveaux statuts. Les réunions sont nombreuses, le travail complexe. Enfin, lors de son Assemblée générale de Québec en 2001, l'association, rebaptisée Agence universitaire de la Francophonie, adopte des statuts qui confirment sa nature d'association de droit québécois, avec un Conseil d'administration de 26 membres, parmi lesquels 11 représentants de 11 des 55 pays membres de l'Organisation internationale de la Francophonie et... 15 recteurs et présidents d'université. Les universitaires conservent la majorité et, avec elle, la possibilité de formuler des contrepropositions, le cas échéant, si les décisions des Sommets des chefs d'État et de gouvernement de la Francophonie leur paraissent insuffisantes à l'égard des difficultés spécifiques que rencontrent leurs établissements. La suite de l'histoire a montré que, si jamais on n'a observé de contradiction entre les décisions prises par les représentants de la Francophonie politique et la dynamique de l'AUF, en revanche, cette stabilisation institutionnelle a permis à l'AUF de se constituer en véritable porte-parole des demandes des universités.

11 Elle a été, en outre, à l'origine d'un élargissement considérable des demandes d'adhésion. De nombreuses universités, constatant que des procédures transparentes étaient mises en place, clairement définies et détaillées dans un Guide des procédures et des programmes, ont en effet souhaité devenir membres de l'AUF. Il convient de plus de souligner que les nouveaux statuts ont largement facilité ce processus d'adhésion: jusqu'en 2001, les universités devaient le plus souvent, pour être admises, appartenir à l'un des pays membres de la Francophonie politique. Depuis la réforme des statuts, toute université, tout centre de recherche, toute grande école qui accueille une filière et délivre un diplôme en langue française peut prétendre à la candidature. Outre le Conseil d'administration et le Conseil scientifique, les statuts adoptés à Québec ont prévu, afin de prendre en charge cette nouvelle procédure d'adhésion, un Conseil dit "associatif ", exclusivement composé d'universitaires. Les dossiers sont étudiés à l'aune de critères comme la compétence universitaire des professeurs, mais aussi leur statut (permanents ou vacataires), ou encore comme la reconnaissance des diplômes de l'institution par l'État qui l'abrite. Nous sommes ainsi passés de 210 universités membres en 1998 à 659 à ce jour.

12 Les établissements membres peuvent être titulaires ou associés. Avec leur adhésion, ils obtiennent des droits strictement identiques en termes de service rendu (bourses de 
mobilité, programmes de coopération scientifique interuniversitaire, pôles d'excellence régionaux, etc.). En revanche, seuls les membres titulaires ont le droit de vote lors de l'Assemblée générale, réunie tous les quatre ans, qui élit les représentants aux Conseils scientifique, associatif et d'administration, ainsi que le Président de l'Agence.

En parallèle à l'instauration de ces règles, l'institution a connu une simplification en termes de programmes. Avant la révision des statuts, le nombre des programmes avait progressivement augmenté, et ceux-ci correspondaient davantage aux différentes formes de contribution des États qu'à des modes d'intervention coordonnés. Avec l'harmonisation de la Francophonie institutionnelle en 2005, l'Agence intergouvernementale de la Francophonie a disparu au profit d'une organisation unique, l'Organisation internationale de la Francophonie. L'adoption d'un plan stratégique décennal, lors du Sommet de Ouagadougou, en 2004, a permis la définition de quatre programmes prioritaires pour l'ensemble de la Francophonie. La traduction de ce nouveau schéma dans l'Agence universitaire de la Francophonie a conduit à la réduction à quatre de ses programmes :

Le programme A concerne la langue française. Il comprend notamment des filières de formation de formateurs en français - intervenant dans toutes les disciplines -, qui sont une spécialité des pays d'Europe de l'Est et d'Asie Pacifique, où elles se développent en nombre croissant. Il regroupe également des réseaux de chercheurs, en particulier de linguistes, mais aussi de littéraires et de spécialistes des métiers de la culture et de la création, c'est-à-dire de disciplines artistiques. Ce programme est considéré comme central dans toutes les institutions liées à la Francophonie institutionnelle.

15 Le programme B s'occupe de démocratie et d'État de droit; il associe les départements de droit de nos universités membres pour faire émerger des recherches portant en particulier sur les variétés de droit francophone, qu'elles soient liées à la Common Law, au droit coutumier ou au droit de source musulmane, qui entrent en contact avec le droit de source francophone et nécessitent des espaces de négociation. Ce programme compte aussi des recherches portant sur la philosophe du droit ou encore le droit de la santé.

Le programme C, dédié à l'éducation, dispose de deux volets. Le premier propose un soutien au renforcement de l'excellence universitaire et de la recherche : il se décline sous la forme de diverses propositions, des projets de coopération scientifique interuniversitaires (PCSI) à la bonne gouvernance, en passant par les pôles d'excellence régionaux ou les bourses. Le second volet est lié à la dimension virtuelle de l'action de l'AUF, laquelle se développe, dans le monde entier, à partir de campus numériques francophones et de centres d'accès à l'information, installations qui permettent l'accès à des dispositifs de visioconférences et d'enseignement en ligne, à des bases de données numérisées, en français ou dans d'autres langues. Ces campus sont extrêmement appréciés, en particulier en Afrique, où ils garantissent notre notoriété. De Nouakchott à Bujumbura en passant par Madagascar, les étudiants tirent profit de ces points d'accès à Internet, et nous développons un nombre croissant de diplômes à distance.

Le programme D concerne, enfin, l'environnement durable. Il regroupe les réseaux de chercheurs qui travaillent sur l'eau, l'aridité, les sols, la réduction des émissions de $\mathrm{CO}_{2}$, les énergies renouvelables... Ces réseaux entretiennent en outre des relations avec le monde des entreprises, avec lequel les partenariats se multiplient. 
18 Aujourd'hui, l'AUF s'investit donc dans ces quatre programmes d'action prioritaires, définis en faveur des universités et pilotés par les universités. Les demandes de nos membres restent en effet notre règle d'action. Grâce au soutien financier accru de la France, nous avons pu ces dernières années stabiliser puis accroître de manière sensible notre budget. Lors du Sommet de la Francophonie, qui s'est réuni à Québec en novembre 2008, les chefs d'Etat et de gouvernement ont affirmé que leur appui, dans le respect des règles propres à l'AUF, ne faiblirait pas.

Institution progressivement construite de manière particulière à l'intérieur d'un ensemble institutionnel plus vaste, l'AUF reste soucieuse de conserver son originalité tout en s'associant avec enthousiasme aux objectifs définis par les Sommets des chefs d'État et de gouvernement de la Francophonie. Elle se donne aujourd'hui comme perspective le renforcement de l'autonomie de ses membres universitaires et de leur solidarité, en continuant de prouver que la science peut s'écrire au Sud comme elle s'écrit au Nord. Le premier cours au monde de droit de l'Internet en français n'est-il pas dispensé, sous l'égide de l'AUF, à l'université Gaston-Berger de Saint-Louis, au Sénégal ?

\section{RÉSUMÉS}

L'histoire de l'AUF, institution universitaire et francophone, est marquée à la fois par l'évolution de la Francophonie institutionnelle et par sa dimension proprement universitaire. C'est au début des années 1960 qu'est fondée l'association des universités partiellement ou entièrement de langue française, l'AUPELF, réseau mis en place par quelques universitaires de divers pays soucieux d'échanges et de coopération. Le caractère associatif de cette instance, qui regroupe rapidement une quarantaine d'universités, permet des prises de décision collégiales et garantit une véritable liberté d'action, mais la maintient dans un état de fragilité financière.

En 1987, l'AUPELF devient l'AUPELF-UREF, par adjonction d'une «université des réseaux d'expression française ", sorte d'université hors les murs, mais sa situation ne s'améliore pas.

Deux événements amènent l'institution à se redresser, à se transformer et à se consolider tout en préservant son autonomie. D'une part, en 1997, avec l'établissement des nouvelles structures de la Francophonie politique, l'AUPELF trouve une nouvelle dynamique : elle devient l'AUF, bras armé universitaire de l'AIF (qui deviendra l'OIF). D'autre part, au début des années 2000, la refonte des statuts et une nouvelle impulsion interne, qui étend les possibilités d'adhésion volontaire des institutions d'enseignement supérieur et de recherche et déconcentre la gestion des programmes, assure à l'AUF l'appui des États membres sans que soit amoindrie sa capacité propre de décision et d'action, garanties par les nouveaux statuts. Un nombre important d'universités font leur entrée dans l'ensemble mondial qui se dessine. Des programmes prioritaires de recherche et d'éducation sont définis : langue et culture francophones, démocratie et État de droit, campus numériques francophones et accès à l'information scientifique, environnement durable. L'évolution qui s'engage donne un rôle important aux initiatives des membres actifs, ce qui conforte la mutation encore en cours.

The history of AUF, an academic and a francophone institution, bears the dual imprint of the development of institutional Francophonie and of its specific academic character. In the early 1960s', AUPELF ('Association of partly or entirely French-speaking universities') - a network set 
by a few international scholars in order to foster exchanges and cooperation - was established. Its status as an association, soon to boast about forty universities, allows AUPELF to make collegial decisions and guarantees its executive freedom while, however, keeping it in financial instability.

In 1987, AUPELF becomes AUPELF-UREF, the latter acronym standing for 'University of Frenchspeaking networks', a kind of extramural university, yet it remains financially fragile.

Two events bring about a recovery, a transformation and a strengthening while preserving its autonomy. First, in 1997, along with the establishment of new structures for political Francophonie, AUPELF finds a new impetus, becoming AUF, the academic strong arm of AIF (which is to become OIF). Then, after the year 2000, thanks to an overhaul of its statutes and a new internal impulse, with less restrictive membership rules for academic and research institutions willing to join and more decentralized management of programmes, AUF receives a support from member states without giving up any of its own ability to make decisions and to implement them. Many universities join the world network which thus takes shape. Priority research and education programmes are defined: francophone language and culture, democracy and rule of law, digital francophone campuses with access to scientific information, sustainable environment. Such a development leaves more room for the initiatives of active members, thus strengthening the ongoing transformation.121-130

\section{INDEX}

Mots-clés : francophonie, institutions, politique, histoire, universités

Keywords : francophony, institutions, policy, history, universities

\section{AUTEUR}

\section{MICHÈLE GENDREAU-MASSALOUX}

Recteur de l'Agence universitaire de la Francophonie

michele.gendreau-massaloux@um-elysee.fr 